\title{
On Simultaneous Approximation of Modified Baskakov-Durrmeyer Operators
}

\author{
Prashantkumar G. Patel ${ }^{1,2}$ and Vishnu Narayan Mishra ${ }^{1,3}$ \\ ${ }^{1}$ Department of Applied Mathematics and Humanities, Sardar Vallabhbhai National Institute of Technology, \\ Ichchhanath Mahadev Dumas Road, Surat, Gujarat 395 007, India \\ ${ }^{2}$ Department of Mathematics, St. Xavier College, Ahmedabad, Gujarat 380 009, India \\ ${ }^{3}$ L. 1627 Awadh Puri Colony Beniganj, Phase-III, Opposite-Industrial Training Institute (I.T.I.), Ayodhya Main Road, Faizabad,
} Uttar Pradesh 224 001, India

Correspondence should be addressed to Prashantkumar G. Patel; prashant225@gmail.com

Received 22 July 2015; Accepted 10 September 2015

Academic Editor: Ying Hu

Copyright (C) 2015 P. G. Patel and V. N. Mishra. This is an open access article distributed under the Creative Commons Attribution License, which permits unrestricted use, distribution, and reproduction in any medium, provided the original work is properly cited.

We discuss properties of modified Baskakov-Durrmeyer-Stancu (BDS) operators with parameter $\gamma>0$. We compute the moments of these modified operators. Also, we establish pointwise convergence, Voronovskaja type asymptotic formula, and an error estimation in terms of second order modification of continuity of the function for the operators $B_{n, \gamma}^{\alpha, \beta}(f, x)$.

\section{Introduction}

For $x \in[0, \infty), \gamma>0,0 \leq \alpha \leq \beta$, and $f \in C[0, \infty)$, we consider a certain integral type generalized Baskakov operators as

$$
\begin{aligned}
B_{n, \gamma}^{\alpha, \beta} & (f(t), x) \\
= & \sum_{k=1}^{\infty} p_{n, k, \gamma}(x) \int_{0}^{\infty} b_{n, k, \gamma}(t) f\left(\frac{n t+\alpha}{n+\beta}\right) d t \\
& +p_{n, 0, \gamma}(x) f\left(\frac{\alpha}{n+\beta}\right) \\
= & \int_{0}^{\infty} W_{n, \gamma}(x, t) f\left(\frac{n t+\alpha}{n+\beta}\right) d t,
\end{aligned}
$$

where

$$
p_{n, k, \gamma}(x)=\frac{\Gamma(n / \gamma+k)}{\Gamma(k+1) \Gamma(n / \gamma)} \cdot \frac{(\gamma x)^{k}}{(1+\gamma x)^{(n / \gamma)+k}},
$$

$$
\begin{aligned}
b_{n, k, \gamma}(t) & =\frac{\gamma \Gamma(n / \gamma+k+1)}{\Gamma(k) \Gamma(n / \gamma+1)} \cdot \frac{(\gamma t)^{k-1}}{(1+\gamma t)^{(n / \gamma)+k+1}}, \\
W_{n, \gamma}(x, t) & =\sum_{k=1}^{\infty} p_{n, k, \gamma}(x) b_{n, k, \gamma}(t)+(1+\gamma x)^{-n / \gamma} \delta(t),
\end{aligned}
$$

$\delta(t)$ being the Dirac delta function.

The operators defined by (1) are the generalization of the integral modification of well-known Baskakov operators having weight function of some beta basis function. As a special case, that is, $\gamma=1$, the operators (1) reduce to the operators very recently studied in $[1,2]$. Inverse results of same type of operators were established in [3]. Also, if $\alpha=\beta=$ 0 , the operators (1) reduce to the operators recently studied in [4] and if $\alpha=\beta=0$ and $\gamma=1$, the operators (1) reduce to the operators studied in [5]. The $q$-analog of the operators (1) is discussed in [6]. We refer to some of the important papers on the recent development on similar type of the operators [7-9]. 
The present a paper that deals with the study of simultaneous approximation for the operators $B_{n, \gamma}^{\alpha, \beta}$.

\section{Moments and Recurrence Relations}

Lemma 1. If one defines the central moments, for every $m \in \mathbb{N}$, as

$$
\begin{aligned}
& \mu_{n, m, \gamma}(x)=B_{n, \gamma}^{\alpha, \beta}\left((t-x)^{m}, x\right) \\
& =\sum_{k=1}^{\infty} p_{n, k, \gamma}(x) \int_{0}^{\infty} b_{n, k, \gamma}(t)\left(\frac{n t+\alpha}{n+\beta}-x\right)^{m} d t \\
& \quad+p_{n, 0, \gamma}(x)\left(\frac{\alpha}{n+\beta}-x\right)^{m},
\end{aligned}
$$

$$
\begin{aligned}
& \cdot \mu_{n, m, \gamma}(x) \\
& +\left\{m \gamma(n+\beta)\left(\frac{\alpha}{n+\beta}-x\right)^{2}-m n\left(\frac{\alpha}{n+\beta}-x\right)\right\} \\
& \cdot \mu_{n, m-1, \gamma}(x) .
\end{aligned}
$$

From the recurrence relation, it can be easily verified that, for all $x \in[0, \infty)$, one has $\mu_{n, m, \gamma}(x)=O\left(n^{-[(m+1) / 2]}\right)$, where $[\alpha]$ denotes the integral part of $\alpha$.

Proof. Taking derivative of the above,

$$
\begin{aligned}
& \mu_{n, m, \gamma}^{(1)}(x)=-m \sum_{k=1}^{\infty} p_{n, k, \gamma}(x) \\
& \cdot \int_{0}^{\infty} b_{n, k, \gamma}(t)\left(\frac{n t+\alpha}{n+\beta}-x\right)^{m-1} d t-m p_{n, 0, \gamma}(x) \\
& \cdot\left(\frac{\alpha}{n+\beta}-x\right)^{m-1}+\sum_{k=1}^{\infty} p_{n, k, \gamma}^{(1)}(x) \\
& \cdot \int_{0}^{\infty} b_{n, k, \gamma}(t)\left(\frac{n t+\alpha}{n+\beta}-x\right)^{m} d t+p_{n, 0, \gamma}^{(1)}(x) \\
& \cdot\left(\frac{\alpha}{n+\beta}-x\right)^{m}=-m \mu_{n, m-1, \gamma}(x)+\sum_{k=1}^{\infty} p_{n, k, \gamma}^{(1)}(x) \\
& \cdot \int_{0}^{\infty} b_{n, k, \gamma}(t)\left(\frac{n t+\alpha}{n+\beta}-x\right)^{m} d t+p_{n, 0, \gamma}^{(1)}(x) \\
& \cdot\left(\frac{\alpha}{n+\beta}-x\right)^{m},
\end{aligned}
$$

$$
\begin{aligned}
x( & +\gamma x)\left\{\mu_{n, m, \gamma}^{(1)}(x)+m \mu_{n, m-1, \gamma}(x)\right\} \\
= & \sum_{k=1}^{\infty} x(1+\gamma x) p_{n, k, \gamma}^{(1)}(x) \\
& \cdot \int_{0}^{\infty} b_{n, k, \gamma}(t)\left(\frac{n t+\alpha}{n+\beta}-x\right)^{m} d t+x(1+\gamma x) \\
& \cdot p_{n, 0, \gamma}^{(1)}(x)\left(\frac{\alpha}{n+\beta}-x\right)^{m} .
\end{aligned}
$$

Using $x(1+\gamma x) p_{n, k, \gamma}^{(1)}(x)=(k-n x) p_{n, k, \gamma}(x)$, we get

$$
\begin{aligned}
x & (1+\gamma x)\left\{\mu_{n, m, \gamma}^{(1)}(x)+m \mu_{n, m-1, \gamma}(x)\right\} \\
= & \sum_{k=1}^{\infty}(k-n x) p_{n, k, \gamma}(x) \\
\cdot & \int_{0}^{\infty} b_{n, k, \gamma}(t)\left(\frac{n t+\alpha}{n+\beta}-x\right)^{m} d t+(-n x) p_{n, 0, \gamma}(x) \\
& \cdot\left(\frac{\alpha}{n+\beta}-x\right)^{m}=\sum_{k=1}^{\infty} k p_{n, k, \gamma}(x) \\
& \cdot \int_{0}^{\infty} b_{n, k, \gamma}(t)\left(\frac{n t+\alpha}{n+\beta}-x\right)^{m} d t-n x \mu_{n, m, \gamma}(x):=I \\
& -n x \mu_{n, m, \gamma}(x) .
\end{aligned}
$$

We can write $I$ as

$$
\begin{aligned}
I= & \left\{\sum_{k=1}^{\infty} p_{n, k, \gamma}(x) \int_{0}^{\infty}\{(k-1)-(n+2 \gamma) t\} b_{n, k, \gamma}(t)\right. \\
& \cdot\left(\frac{n t+\alpha}{n+\beta}-x\right)^{m} d t+\sum_{k=1}^{\infty} p_{n, k, \gamma}(x) \int_{0}^{\infty} b_{n, k, \gamma}(t) \\
& \left.\cdot\left(\frac{n t+\alpha}{n+\beta}-x\right)^{m} d t\right\}+\left\{(n+2 \gamma) \sum_{k=1}^{\infty} p_{n, k, \gamma}(x)\right. \\
& \left.\cdot \int_{0}^{\infty} t b_{n, k, \gamma}(t)\left(\frac{n t+\alpha}{n+\beta}-x\right)^{m} d t\right\}:=I_{1} \\
& +I_{2} \text { (say). }
\end{aligned}
$$

To estimate $I_{2}$ using $t=((n+\beta) / n)\{((n t+\alpha) /(n+\beta)-x)-$ $(\alpha /(n+\beta)-x)\}$, we have

$$
\begin{aligned}
I_{2} & =\frac{(n+2 \gamma)(n+\beta)}{n}\left\{\sum_{k=1}^{\infty} p_{n, k, \gamma}(x)\right. \\
& \cdot \int_{0}^{\infty} b_{n, k, \gamma}(t)\left(\frac{n t+\alpha}{n+\beta}-x\right)^{m+1} d t-\left(\frac{\alpha}{n+\beta}\right. \\
& \left.-x) \sum_{k=1}^{\infty} p_{n, k, \gamma}(x) \int_{0}^{\infty} b_{n, k, \gamma}(t)\left(\frac{n t+\alpha}{n+\beta}-x\right)^{m} d t\right\}
\end{aligned}
$$


International Journal of Analysis

3

$$
\begin{aligned}
& =\frac{(n+2 \gamma)(n+\beta)}{n}\left\{\sum_{k=1}^{\infty} p_{n, k, \gamma}(x)\right. \\
& \cdot \int_{0}^{\infty} b_{n, k, \gamma}(t)\left(\frac{n t+\alpha}{n+\beta}-x\right)^{m+1} d t+p_{n, 0, \gamma}(x) \\
& \cdot\left(\frac{\alpha}{n+\beta}-x\right)^{m+1}-\left(\frac{\alpha}{n+\beta}-x\right) \\
& \cdot\left\{\sum_{k=1}^{\infty} p_{n, k, \gamma}(x) \int_{0}^{\infty} b_{n, k, \gamma}(t)\left(\frac{n t+\alpha}{n+\beta}-x\right)^{m} d t\right. \\
& \left.\left.+p_{n, 0, \gamma}(x)\left(\frac{\alpha}{n+\beta}-x\right)^{m}\right\}\right\} \\
& =\frac{(n+2 \gamma)(n+\beta)}{n}\left\{\mu_{n, m+1, \gamma}(x)-\left(\frac{\alpha}{n+\beta}-x\right)\right. \\
& \left.\cdot \mu_{n, m, \gamma}(x)\right\} \cdot
\end{aligned}
$$

Next, to estimate $I_{1}$ using the equality, $\{(k-1)-(n+$ $2 \gamma) t\} b_{n, k, \gamma}(t)=t(1+\gamma t) b_{n, k, \gamma}^{(1)}(t)$, we have

$$
\begin{aligned}
I_{1} & =\left\{\sum_{k=1}^{\infty} p_{n, k, \gamma}(x) \int_{0}^{\infty} t b_{n, k, \gamma}^{(1)}(t)\left(\frac{n t+\alpha}{n+\beta}-x\right)^{m} d t\right. \\
& \left.+\sum_{k=1}^{\infty} p_{n, k, \gamma}(x) \int_{0}^{\infty} b_{n, k, \gamma}(t)\left(\frac{n t+\alpha}{n+\beta}-x\right)^{m} d t\right\} \\
& +\gamma \sum_{k=1}^{\infty} p_{n, k, \gamma}(x) \int_{0}^{\infty} t^{2} b_{n, k, \gamma}^{(1)}(t)\left(\frac{n t+\alpha}{n+\beta}-x\right)^{m} d t \\
& :=J_{1}+J_{2} \text { (say). }
\end{aligned}
$$

Again putting $t=((n+\beta) / n)\{((n t+\alpha) /(n+\beta)-x)-(\alpha /(n+$ $\beta)-x)\}$, we get

$$
\begin{aligned}
J_{1} & =\frac{n+\beta}{n}\left\{\sum_{k=1}^{\infty} p_{n, k, \gamma}(x)\right. \\
& \cdot \int_{0}^{\infty} b_{n, k, \gamma}^{(1)}(t)\left(\frac{n t+\alpha}{n+\beta}-x\right)^{m+1} d t+\left(\frac{\alpha}{n+\beta}-x\right) \\
& \left.\cdot \sum_{k=1}^{\infty} p_{n, k, \gamma}(x) \int_{0}^{\infty} b_{n, k, \gamma}^{(1)}(t)\left(\frac{n t+\alpha}{n+\beta}-x\right)^{m} d t\right\} \\
& +\sum_{k=1}^{\infty} p_{n, k, \gamma}(x) \int_{0}^{\infty} b_{n, k, \gamma}(t)\left(\frac{n t+\alpha}{n+\beta}-x\right)^{m} d t .
\end{aligned}
$$

Now, integrating by parts, we get

$$
\begin{aligned}
& J_{1}=-(m+1) \sum_{k=1}^{\infty} p_{n, k, \gamma}(x) \\
& \cdot \int_{0}^{\infty} b_{n, k, \gamma}(t)\left(\frac{n t+\alpha}{n+\beta}-x\right)^{m} d t+m\left(\frac{\alpha}{n+\beta}-x\right) \\
& \cdot \sum_{k=1}^{\infty} p_{n, k, \gamma}(x) \int_{0}^{\infty} b_{n, k, \gamma}(t)\left(\frac{n t+\alpha}{n+\beta}-x\right)^{m-1} d t \\
& +\sum_{k=1}^{\infty} p_{n, k, \gamma}(x) \int_{0}^{\infty} b_{n, k, \gamma}(t)\left(\frac{n t+\alpha}{n+\beta}-x\right)^{m} d t \\
& =-(m+1) \\
& \left\{\sum_{k=1}^{\infty} p_{n, k, \gamma}(x) \int_{0}^{\infty} b_{n, k, \gamma}(t)\left(\frac{n t+\alpha}{n+\beta}-x\right)^{m} d t\right. \\
& \left.+p_{n, 0, \gamma}(x)\left(\frac{\alpha}{n+\beta}-x\right)^{m}\right\}+m\left(\frac{\alpha}{n+\beta}-x\right) \\
& \cdot\left\{\sum_{k=1}^{\infty} p_{n, k, \gamma}(x) \int_{0}^{\infty} b_{n, k, \gamma}(t)\left(\frac{n t+\alpha}{n+\beta}-x\right)^{m-1} d t\right. \\
& \left.+p_{n, 0, \gamma}(x)\left(\frac{\alpha}{n+\beta}-x\right)^{m-1}\right\}+\sum_{k=1}^{\infty} p_{n, k, \gamma}(x) \\
& \cdot \int_{0}^{\infty} b_{n, k, \gamma}(t)\left(\frac{n t+\alpha}{n+\beta}-x\right)^{m} d t+p_{n, 0, \gamma}(x)\left(\frac{\alpha}{n+\beta}\right. \\
& -x)^{m} \\
& J_{1}=-m \mu_{n, m, \gamma}(x)+m\left(\frac{\alpha}{n+\beta}-x\right) \mu_{n, m-1, \gamma}(x) .
\end{aligned}
$$

Proceeding in the similar manner, we obtain the estimate $J_{2}$ as

$$
\begin{aligned}
J_{2}= & -\frac{\gamma(n+\beta)(m+2)}{n} \mu_{n, m+1, \gamma}(x) \\
& +2 \gamma \frac{(n+\beta)(m+1)}{n}\left(\frac{\alpha}{n+\beta}-x\right) \mu_{n, m, \gamma}(x) \\
& -\frac{m \gamma(n+\beta)}{n}\left(\frac{\alpha}{n+\beta}-x\right)^{2} \mu_{n, m-1, \gamma}(x) .
\end{aligned}
$$

Combining (6)-(12), we get

$$
\begin{array}{r}
x(1+\gamma x)\left\{\mu_{n, m, \gamma}^{(1)}(x)+m \mu_{n, m-1, \gamma}(x)\right\}=-m \mu_{n, m, \gamma}(x) \\
+m\left(\frac{\alpha}{n+\beta}-x\right) \mu_{n, m-1, \gamma}(x)-\frac{\gamma(n+\beta)(m+2)}{n} \\
\cdot \mu_{n, m+1, \gamma}(x)+2 \gamma \frac{(n+\beta)(m+1)}{n}\left(\frac{\alpha}{n+\beta}-x\right)
\end{array}
$$




$$
\begin{aligned}
& \cdot \mu_{n, m, \gamma}(x)-\frac{m \gamma(n+\beta)}{n}\left(\frac{\alpha}{n+\beta}-x\right)^{2} \\
& \cdot \mu_{n, m-1, \gamma}(x)-n x \mu_{n, m, \gamma}(x) \\
& +\frac{(n+2 \gamma)(n+\beta)}{n}\left\{\mu_{n, m+1, \gamma}(x)\right. \\
& \left.-\left(\frac{\alpha}{n+\beta}-x\right) \mu_{n, m, \gamma}(x)\right\} .
\end{aligned}
$$

Hence,

$$
\begin{aligned}
& (n-\gamma m)(n+\beta) \mu_{n, m+1, \gamma}(x)=n x(1+\gamma x)\left\{\mu_{n, m, \gamma}^{(1)}(x)\right. \\
& \left.\quad+m \mu_{n, m-1, \gamma}(x)\right\}+\left\{m n+n^{2} x\right. \\
& \quad-(2 \gamma m-n)(\alpha-(n+\beta) x)\} \mu_{n, m, \gamma}(x) \\
& \quad+\left\{m \gamma(n+\beta)\left(\frac{\alpha}{n+\beta}-x\right)^{2}\right. \\
& \left.\quad-m n\left(\frac{\alpha}{n+\beta}-x\right)\right\} \mu_{n, m-1, \gamma}(x) .
\end{aligned}
$$

This completes the proof of Lemma 1.

Remark 2 (see [10]). For $m \in \mathbb{N} \cup\{0\}$, if the $m$ th order moment is defined as

$$
U_{n, m, \gamma}(x)=\sum_{k=0}^{\infty} p_{n, k, \gamma}(x)\left(\frac{k}{n}-x\right)^{m},
$$

then $U_{n, 0, \gamma}(x)=1, U_{n, 1, \gamma}(x)=0$, and $n U_{n, m+1, \gamma}(x)=x(1+$ $\gamma x)\left(U_{n, m, \gamma}^{(1)}(x)+m U_{n, m-1, \gamma}(x)\right)$.

Consequently, for all $x \in[0, \infty)$, we have $U_{n, m, \gamma}(x)=$ $O\left(n^{-[(m+1) / 2]}\right)$.

Remark 3. It is easily verified from Lemma 1 that for each $x \in$ $[0, \infty)$

$$
\begin{aligned}
& B_{n, \gamma}^{\alpha, \beta}\left(t^{m}, x\right)=\frac{n^{m} \Gamma(n / \gamma+m) \Gamma(n / \gamma-m+1)}{(n+\beta)^{m} \Gamma(n / \gamma+1) \Gamma(n / \gamma)} x^{m} \\
& +\frac{m n^{m-1} \Gamma(n / \gamma+m-1) \Gamma(n / \gamma-m+1)}{(n+\beta)^{m} \Gamma(n / \gamma+1) \Gamma(n / \gamma)}\{n(m-1) \\
& \left.+\alpha\left(\frac{n}{\gamma}-m+1\right)\right\} x^{m-1} \\
& +\frac{\alpha m(m-1) n^{m-2} \Gamma(n / \gamma+m-2) \Gamma(n / \gamma-m+2)}{(n+\beta)^{m} \Gamma(n / \gamma+1) \Gamma(n / \gamma)}\{n(m \\
& \left.-2)+\frac{\alpha(n / \gamma-m+2)}{2}\right\} x^{m-2}+O\left(n^{-2}\right) .
\end{aligned}
$$

Lemma 4 (see [10]). The polynomials $Q_{i, j, r, \gamma}(x)$ exist independent of $n$ and $k$ such that

$$
\begin{aligned}
& \left\{x(1+\gamma x)^{r}\right\} D^{r}\left[p_{n, k, \gamma}(x)\right] \\
& =\sum_{\substack{2 i+j \leq r \\
i, j \geq 0}} n^{i}(k-n x)^{j} Q_{i, j, r, \gamma}(x) p_{n, k, \gamma}(x), \\
& \text { where } D \equiv \frac{d}{d x} .
\end{aligned}
$$

Lemma 5. If $f$ is $r$ times differentiable on $[0, \infty)$, such that $f^{(r-1)}=O\left(t^{v}\right), v>0$ as $t \rightarrow \infty$, then for $r=1,2,3, \ldots$ and $n>v+\gamma r$ one has

$$
\begin{aligned}
& \left(B_{n, \gamma}^{\alpha, \beta}\right)^{(r)}(f, x)=\frac{n^{r} \Gamma(n / \gamma+r) \Gamma(n / \gamma-r+1)}{(n+\beta)^{r} \Gamma(n / \gamma+1) \Gamma(n / \gamma)} \\
& \cdot \sum_{k=0}^{\infty} p_{n+\gamma r, k, \gamma}(x) \\
& \cdot \int_{0}^{\infty} b_{n-\gamma r, k+r, \gamma}(t) f^{(r)}\left(\frac{n t+\alpha}{n+\beta}\right) d t .
\end{aligned}
$$

Proof. First

$$
\begin{aligned}
& \left(B_{n, \gamma}^{\alpha, \beta}\right)^{(1)}(f, x) \\
& =\sum_{k=1}^{\infty} p_{n, k, \gamma}^{(1)}(x) \int_{0}^{\infty} b_{n, k, \gamma}(t) f\left(\frac{n t+\alpha}{n+\beta}\right) d t \\
& \quad-n(1+\gamma x)^{-n / \gamma-1} f\left(\frac{\alpha}{n+\beta}\right) .
\end{aligned}
$$

Now, using the identities

$$
\begin{aligned}
& p_{n, k, \gamma}^{(1)}(x)=n\left\{p_{n+\gamma, k-1, \gamma}(x)-p_{n+\gamma, k, \gamma}(x)\right\}, \\
& b_{n, k, \gamma}^{(1)}(x)=(n+\gamma)\left\{b_{n+\gamma, k-1, \gamma}(x)-b_{n+\gamma, k, \gamma}(x)\right\},
\end{aligned}
$$

for $k \geq 1$, we have

$$
\begin{aligned}
& \left(B_{n, \gamma}^{\alpha, \beta}\right)^{(1)}(f, x)=\sum_{k=1}^{\infty} n\left\{p_{n+\gamma, k-1, \gamma}(x)-p_{n+\gamma, k, \gamma}(x)\right\} \\
& \cdot \int_{0}^{\infty} b_{n, k, \gamma}(t) f\left(\frac{n t+\alpha}{n+\beta}\right) d t-n(1+\gamma x)^{-n / \gamma-1} \\
& \cdot f\left(\frac{\alpha}{n+\beta}\right)=n p_{n+\gamma, 0, \gamma}(x) \\
& \cdot \int_{0}^{\infty} b_{n+\gamma, 1, \gamma}(t) f\left(\frac{n t+\alpha}{n+\beta}\right) d t-n(1+\gamma x)^{-n / \gamma-1} \\
& \cdot f\left(\frac{\alpha}{n+\beta}\right)+n \sum_{k=1}^{\infty} p_{n+\gamma, k, \gamma}(x) \\
& \cdot \int_{0}^{\infty}\left\{b_{n, k+1, \gamma}(t)-b_{n, k, \gamma}(t)\right\} f\left(\frac{n t+\alpha}{n+\beta}\right) d t,
\end{aligned}
$$




$$
\begin{aligned}
& \left(B_{n, \gamma}^{\alpha, \beta}\right)^{(1)}(f, x)=n(1+\gamma x)^{-n / \gamma-1} \\
& \cdot \int_{0}^{\infty}(n+\gamma)(1+\gamma t)^{-n / \gamma-2} f\left(\frac{n t+\alpha}{n+\beta}\right) d t \\
& +n \sum_{k=1}^{\infty} p_{n+\gamma, k, \gamma}(x) \\
& \cdot \int_{0}^{\infty}\left(-\frac{1}{n} b_{n-\gamma, k+1, \gamma}^{(1)}(t)\right) f\left(\frac{n t+\alpha}{n+\beta}\right) d t \\
& -n(1+\gamma x)^{-n / \gamma-1} f\left(\frac{\alpha}{n+\beta}\right) .
\end{aligned}
$$

Integrating by parts, we get

$$
\begin{aligned}
& \left(B_{n, \gamma}^{\alpha, \beta}\right)^{(1)}(f, x)=n(1+\gamma x)^{-n / \gamma-1} f\left(\frac{\alpha}{n+\beta}\right) \\
& +\frac{n^{2}}{n+\beta}(1+\gamma x)^{-n / \gamma-1} \\
& \cdot \int_{0}^{\infty}(1+\gamma t)^{-n / \gamma-1} f^{(1)}\left(\frac{n t+\alpha}{n+\beta}\right) d t+\frac{n}{n+\beta} \\
& \cdot \sum_{k=1}^{\infty} p_{n+\gamma, k, \gamma}(x) \int_{0}^{\infty} b_{n-\gamma, k+1, \gamma}(t) f^{(1)}\left(\frac{n t+\alpha}{n+\beta}\right) d t \\
& -n(1+\gamma x)^{-n / \gamma-1} f\left(\frac{\alpha}{n+\beta}\right), \\
& \left(B_{n, \gamma}^{\alpha, \beta}\right)^{(1)}(f, x)=\frac{n}{n+\beta} \sum_{k=0}^{\infty} p_{n+\gamma, k, \gamma}(x) \\
& \cdot \int_{0}^{\infty} b_{n-\gamma, k+1, \gamma}(t) f^{(1)}\left(\frac{n t+\alpha}{n+\beta}\right) d t .
\end{aligned}
$$

Thus the result is true for $r=1$. We prove the result by induction method. Suppose that the result is true for $r=i$; then

$$
\begin{aligned}
& \left(B_{n, \gamma}^{\alpha, \beta}\right)^{(i)}(f, x)=\frac{n^{i} \Gamma(n / \gamma+i) \Gamma(n / \gamma-i+1)}{(n+\beta)^{i} \Gamma(n / \gamma+1) \Gamma(n / \gamma)} \\
& \cdot \sum_{k=0}^{\infty} p_{n+\gamma i, k, \gamma}(x) \int_{0}^{\infty} b_{n-\gamma i, k+i, \gamma}(t) f^{(i)}\left(\frac{n t+\alpha}{n+\beta}\right) d t .
\end{aligned}
$$

Thus, using the identities (20), we have

$$
\begin{aligned}
\left(B_{n, \gamma}^{\alpha, \beta}\right)^{(i+1)}(f, x) & \\
= & \frac{n^{i} \Gamma(n / \gamma+i) \Gamma(n / \gamma-i+1)}{(n+\beta)^{i} \Gamma(n / \gamma+1) \Gamma(n / \gamma)}\left\{\sum_{k=1}^{\infty}\left(\frac{n}{\gamma}+i\right)\right. \\
& \cdot\left\{p_{n+\gamma(i+1), k-1, \gamma}(x)-p_{n+\gamma(i+1), k, \gamma}(x)\right\} \int_{0}^{\infty} b_{n-\gamma i, k+i, \gamma}(t)
\end{aligned}
$$

$$
\begin{aligned}
& \cdot f^{(i)}\left(\frac{n t+\alpha}{n+\beta}\right) d t-\left(\frac{n}{\gamma}+i\right)(1+\gamma x)^{-n / \gamma-i-1} \\
& \left.\int_{0}^{\infty} b_{n-\gamma i, i, \gamma}(t) f^{(i)}\left(\frac{n t+\alpha}{n+\beta}\right)\right\} \\
& =\frac{n^{i} \Gamma(n / \gamma+i+1) \Gamma(n / \gamma-i+1)}{(n+\beta)^{i} \Gamma(n / \gamma+1) \Gamma(n / \gamma)} p_{n+\gamma(i+1), 0, \gamma}(x) \\
& \cdot \int_{0}^{\infty} b_{n-\gamma i, 1+i, \gamma}(t) f^{(i)}\left(\frac{n t+\alpha}{n+\beta}\right) d t \\
& -\frac{n^{i} \Gamma(n / \gamma+i+1) \Gamma(n / \gamma-i+1)}{(n+\beta)^{i} \Gamma(n / \gamma+1) \Gamma(n / \gamma)} p_{n+\gamma(i+1), 0, \gamma}(x) \\
& \cdot \int_{0}^{\infty} b_{n-\gamma i, i, \gamma}(t) f^{(i)}\left(\frac{n t+\alpha}{n+\beta}\right) d t \\
& +\frac{n^{i} \Gamma(n / \gamma+i+1) \Gamma(n / \gamma-i+1)}{(n+\beta)^{i} \Gamma(n / \gamma+1) \Gamma(n / \gamma)}\left\{\sum_{k=1}^{\infty} p_{n+\gamma(i+1), k, \gamma}(x)\right. \\
& \cdot \int_{0}^{\infty}\left\{b_{n-\gamma i, k+i+1, \gamma}(t)-b_{n-\gamma i, k+i, \gamma}(t)\right\} \\
& \left.f^{(i)}\left(\frac{n t+\alpha}{n+\beta}\right) d t\right\} \\
& =\frac{n^{i} \Gamma(n / \gamma+i+1) \Gamma(n / \gamma-i+1)}{(n+\beta)^{i} \Gamma(n / \gamma+1) \Gamma(n / \gamma)} p_{n+\gamma(i+1), 0, \gamma}(x) \\
& \cdot \int_{0}^{\infty}\left(-\frac{1}{n / \gamma-i} b_{n-\gamma(i-1), 1+i, \gamma}^{(1)}(t)\right) f^{(i)}\left(\frac{n t+\alpha}{n+\beta}\right) d t \\
& +\frac{n^{i} \Gamma(n / \gamma+i+1) \Gamma(n / \gamma-i+1)}{(n+\beta)^{i} \Gamma(n / \gamma+1) \Gamma(n / \gamma)} \sum_{k=1}^{\infty} p_{n+\gamma(i+1), k, \gamma}(x) \\
& \cdot \int_{0}^{\infty}\left(-\frac{1}{n / \gamma-i} b_{n-\gamma(i-1), k+i+1, \gamma}^{(1)}(t)\right) f^{(i)}\left(\frac{n t+\alpha}{n+\beta}\right) d t .
\end{aligned}
$$

Integrating by parts, we obtain

$$
\begin{aligned}
& \left(B_{n, \gamma}^{\alpha, \beta}\right)^{(i+1)}(f, x)=\frac{n^{i+1} \Gamma(n / \gamma+i+1) \Gamma(n / \gamma-i+1)}{(n+\beta)^{i+1} \Gamma(n / \gamma+1) \Gamma(n / \gamma)} \\
& \quad \cdot \sum_{k=0}^{\infty} p_{n+\gamma(i+1), k, \gamma}(x) \\
& \quad . \int_{0}^{\infty} b_{n-\gamma(i-1), k+i+1, \gamma}(t) f^{(i+1)}\left(\frac{n t+\alpha}{n+\beta}\right) d t .
\end{aligned}
$$

This completes the proof of Lemma 5.

\section{Direct Theorems}

This section deals with the direct results; we establish here pointwise approximation, asymptotic formula, and error estimation in simultaneous approximation. 
We denote $C_{\mu}[0, \infty)=\{f \in C[0, \infty):|f(t)| \leq$ $M t^{\mu}$ for some $\left.M>0, \mu>0\right\}$, and the norm $\|\cdot\|_{\mu}$ on the class $C_{\mu}[0, \infty)$ is defined as $\|f\|_{\mu}=\sup _{0 \leq t<\infty}|f(t)| t^{-\mu}$. It can be easily verified that the operators $B_{n, \gamma}^{\alpha, \beta}(f, x)$ are well defined for $f \in C_{\mu}[0, \infty)$.

Theorem 6. Let $f \in C_{\mu}[0, \infty)$ and let $f^{(r)}$ exist at a point $x \in(0, \infty)$. Then one has

$$
\lim _{n \rightarrow \infty}\left(B_{n, \gamma}^{\alpha, \beta}\right)^{(r)}(f, x)=f^{(r)}(x) .
$$

Proof. By Taylor's expansion of $f$, we have

$$
f(t)=\sum_{i=0}^{r} \frac{f^{(i)}(x)}{i !}(t-x)^{i}+\epsilon(t, x)(t-x)^{r},
$$

where $\epsilon(t, x) \rightarrow 0$ as $t \rightarrow x$. Hence,

$$
\begin{aligned}
\left(B_{n, \gamma}^{\alpha, \beta}\right)^{(r)}(f, x)= & \sum_{i=0}^{r} \frac{f^{(i)}(x)}{i !}\left(B_{n, \gamma}^{\alpha, \beta}\right)^{(r)}\left((t-x)^{i}, x\right) \\
& +\left(B_{n, \gamma}^{\alpha, \beta}\right)^{(r)}\left(\epsilon(t, x)(t-x)^{r}, x\right) \\
:= & R_{1}+R_{2} .
\end{aligned}
$$

First, to estimate $R_{1}$, using binomial expansion of $((n t+$ $\alpha) /(n+\beta)-x)^{i}$ and Remark 3, we have

$$
\begin{aligned}
R_{1} & =\sum_{i=0}^{r} \frac{f^{(i)}(x)}{i !} \sum_{j=0}^{i}\left(\begin{array}{c}
i \\
j
\end{array}\right)(-x)^{i-j}\left(B_{n, \gamma}^{\alpha, \beta}\right)^{(r)}\left(t^{j}, x\right) \\
& =\frac{f^{(r)}(x)}{r !}\left\{\frac{n^{r} \Gamma(n / \gamma+r) \Gamma(n / \gamma-r+1)}{(n+\beta)^{r} \Gamma(n / \gamma+1) \Gamma(n / \gamma)} r !\right\} \\
& =f^{(r)}(x)\left\{\frac{n^{r} \Gamma(n / \gamma+r) \Gamma(n / \gamma-r+1)}{(n+\beta)^{r} \Gamma(n / \gamma+1) \Gamma(n / \gamma)}\right\} \\
& \longrightarrow f^{(r)}(x) \quad \text { as } n \longrightarrow \infty .
\end{aligned}
$$

Next, applying Lemma 4, we obtain

$$
\begin{aligned}
R_{2} & =\int_{0}^{\infty} W_{n, \gamma}^{(r)}(t, x) \epsilon(t, x)\left(\frac{n t+\alpha}{n+\beta}-x\right)^{r} d t, \\
\left|R_{2}\right| & \leq \sum_{\substack{2 i+j \leq r \\
i, j \geq 0}} n^{i} \frac{\left|Q_{i, j, r, \gamma}(x)\right|}{\{x(1+\gamma x)\}^{r}} \sum_{k=1}^{\infty}|k-n x|^{j} p_{n, k, \gamma}(x) \\
& \cdot \int_{0}^{\infty} b_{n, k, \gamma}(t)|\epsilon(t, x)| \frac{n t+\alpha}{n+\beta}-\left.x\right|^{r} d t \\
& +\frac{\Gamma(n / \gamma+r+2)}{\Gamma(n / \gamma)}(1+\gamma x)^{-n / \gamma-r}|\epsilon(0, x)| \\
& \cdot\left|\frac{\alpha}{n+\beta}-x\right|^{r} .
\end{aligned}
$$

The second term in the above expression tends to zero as $n \rightarrow$ $\infty$. Since $\epsilon(t, x) \rightarrow 0$ as $t \rightarrow x$ for given $\varepsilon>0$, there exists a $\delta \in(0,1)$ such that $|\epsilon(t, x)|<\varepsilon$ whenever $0<|t-x|<\delta$. If $\tau>\max \{\mu, r\}$, where $\tau$ is any integer, then we can find a constant $M_{3}>0$, such that $\left|\epsilon(t, x)((n t+\alpha) /(n+\beta)-x)^{r}\right| \leq$ $M_{3}|(n t+\alpha) /(n+\beta)-x|^{\tau}$ for $|t-x| \geq \delta$. Therefore,

$$
\begin{aligned}
& \left|R_{2}\right| \leq M_{3} \sum_{\substack{2 i+j \leq r \\
i, j \geq 0}} n^{i} \sum_{k=0}^{\infty}|k-n x|^{j} p_{n, k, \gamma}(x) \\
& \cdot\left\{\varepsilon \int_{|t-x|<\delta} b_{n, k, \gamma}(x)\left|\frac{n t+\alpha}{n+\beta}-x\right|^{r} d t\right. \\
& \left.+\int_{|t-x| \geq \delta} b_{n, k, \gamma}(t)\left|\frac{n t+\alpha}{n+\beta}-x\right|^{\tau} d t\right\}=: R_{3}+R_{4} .
\end{aligned}
$$

Applying the Cauchy-Schwarz inequality for integration and summation, respectively, we obtain

$$
\begin{aligned}
&\left|R_{3}\right| \leq \varepsilon M_{3} \sum_{\substack{2 i+j \leq r \\
i, j \geq 0}} n^{i}\left\{\sum_{k=1}^{\infty}(k-n x)^{2 j} p_{n, k, \gamma}(x)\right\}^{1 / 2} \\
& \cdot\left\{\sum_{k=1}^{\infty} p_{n, k, \gamma}(x) \int_{0}^{\infty} b_{n, k, \gamma}(t)\left(\frac{n t+\alpha}{n+\beta}-x\right)^{2 r} d t\right\}^{1 / 2} .
\end{aligned}
$$

Using Remark 2 and Lemma 1 , we get $R_{3} \leq \varepsilon O\left(n^{r / 2}\right) O\left(n^{-r / 2}\right)$ $=\varepsilon \cdot O(1)$.

Again using the Cauchy-Schwarz inequality and Lemma 1 , we get

$$
\begin{aligned}
& \left|R_{4}\right| \leq M_{4} \sum_{\substack{2 i+j \leq r \\
i, j \geq 0}} n^{i} \sum_{k=1}^{\infty}|k-n x|^{j} p_{n, k, \gamma}(x) \\
& \cdot \int_{|t-x| \geq \delta} b_{n, k, \gamma}(t)\left|\frac{n t+\alpha}{n+\beta}-x\right|^{\tau} d t \leq M_{4} \sum_{\substack{2 i+j \leq r \\
i, j \geq 0}} n^{i} \\
& \cdot \sum_{k=1}^{\infty}|k-n x|^{j} p_{n, k, \gamma}(x)\left\{\int_{|t-x| \geq \delta} b_{n, k, \gamma}(t) d t\right\}^{1 / 2} \\
& \cdot\left\{\int_{|t-x| \geq \delta} b_{n, k, \gamma}(t)\left(\frac{n t+\alpha}{n+\beta}-x\right)^{2 \tau} d t\right\}^{1 / 2} \\
& \leq M_{4} \sum_{\substack{2 i+j \leq r \\
i, j \geq 0}} n^{i}\left\{\sum_{k=1}^{\infty}(k-n x)^{2 j} p_{n, k, \gamma}(x)\right\}^{1 / 2} \\
& \cdot\left\{\sum_{k=1}^{\infty} p_{n, k, \gamma}(x) \int_{0}^{\infty} b_{n, k, \gamma}(t)\left(\frac{n t+\alpha}{n+\beta}-x\right)^{2 \tau} d t\right\} \\
& =\sum_{2 i+j \leq r} n^{i} O\left(n^{j / 2}\right) O\left(n^{-\tau / 2}\right)=O\left(n^{(r-\tau) / 2}\right)=o(1) .
\end{aligned}
$$

Collecting the estimation of $R_{1}-R_{4}$, we get the required result. 
Theorem 7. Let $f \in C_{\mu}[0, \infty)$. If $f^{(r+2)}$ exists at a point $x \in$ $(0, \infty)$, then

$$
\begin{aligned}
\lim _{n \rightarrow \infty} n & \left\{\left(B_{n, \gamma}^{\alpha, \beta}\right)^{(r)}(f, x)-f^{(r)}(x)\right\} \\
= & r(\gamma(r-1)-\beta) f^{(r)}(x) \\
& +\{r \gamma(1+2 x)+\alpha-\beta x\} f^{(r+1)}(x) \\
& +x(1+\gamma x) f^{(r+2)}(x) .
\end{aligned}
$$

Proof. Using Taylor's expansion of $f$, we have

$$
f(t)=\sum_{i=0}^{r+2} \frac{f^{(i)}(x)}{i !}(t-x)^{i}+\epsilon(t, x)(t-x)^{r+2},
$$

where $\epsilon(t, x) \rightarrow 0$ as $t \rightarrow x$ and $\epsilon(t, x)=O\left((t-x)^{\mu}\right), t \rightarrow$ $\infty$ for $\mu>0$.

Applying Lemma 1, we have

$$
\begin{aligned}
& n\left\{\left(B_{n, \gamma}^{\alpha, \beta}\right)^{(r)}(f, x)-f^{(r)}(x)\right\} \\
&=n\left\{\sum_{i=0}^{r+2} \frac{f^{(i)}(x)}{i !}\left(B_{n, \gamma}^{\alpha, \beta}\right)^{(r)}\left((t-x)^{i}, x\right)-f^{(r)}(x)\right\} \\
&+n\left\{\left(B_{n, \gamma}^{\alpha, \beta}\right)^{(r)}\left(\epsilon(t, x)(t-x)^{r+2}, x\right)\right\} \\
&:= E_{1}+E_{2} .
\end{aligned}
$$

First, we have

$$
\begin{aligned}
E_{1} & =n \sum_{i=0}^{r+2} \frac{f^{(i)}(x)}{i !} \sum_{j=0}^{i}\left(\begin{array}{l}
i \\
j
\end{array}\right)(-x)^{i-j}\left(B_{n, \gamma}^{\alpha, \beta}\right)^{(r)}\left(t^{j}, x\right) \\
& -n f^{(r)}(x)=\frac{f^{(r)}(x)}{r !} n\left\{\left(B_{n, \gamma}^{\alpha, \beta}\right)^{(r)}\left(t^{r}, x\right)-r !\right\} \\
& +\frac{f^{(r+1)}(x)}{(r+1) !} n\left\{(r+1)(-x)\left(B_{n, \gamma}^{\alpha, \beta}\right)^{(r)}\left(t^{r}, x\right)\right. \\
& \left.+\left(B_{n, \gamma}^{\alpha, \beta}\right)^{(r)}\left(t^{r+1}, x\right)\right\}+\frac{f^{(r+2)}(x)}{(r+2) !} \\
& \cdot n\left\{\frac{(r+2)(r+1)}{2} x^{2}\left(B_{n, \gamma}^{\alpha, \beta}\right)^{(r)}\left(t^{r}, x\right)+(r+2)\right. \\
& \left.\cdot(-x)\left(B_{n, \gamma}^{\alpha, \beta}\right)^{(r)}\left(t^{r+1}, x\right)+\left(B_{n, \gamma}^{\alpha, \beta}\right)^{(r)}\left(t^{r+2}, x\right)\right\} \\
& =f^{(r)}(x) n\left\{\frac{n^{r} \Gamma(n / \gamma+r) \Gamma(n / \gamma-r+1)}{(n+\beta)^{r} \Gamma(n / \gamma+1) \Gamma(n / \gamma)}-1\right\} \\
& +\frac{f^{(r+1)}(x)}{(r+1) !} n\{(r+1)(-x) \\
& \cdot \frac{n^{r} \Gamma(n / \gamma+r) \Gamma(n / \gamma-r+1)}{(n+\beta)^{r} \Gamma(n / \gamma+1) \Gamma(n / \gamma)} r ! \\
& +\frac{n^{r+1} \Gamma(n / \gamma+r+1) \Gamma(n / \gamma-r)}{(n+\beta)^{r+1} \Gamma(n / \gamma+1) \Gamma(n / \gamma)}(r+1) ! x
\end{aligned}
$$

$$
\begin{aligned}
& +\frac{(r+1) n^{r} \Gamma(n / \gamma+r) \Gamma(n / \gamma-r)}{(n+\beta)^{r+1} \Gamma(n / \gamma+1) \Gamma(n / \gamma)}\left\{n r+\alpha\left(\frac{n}{\gamma}\right.\right. \\
& -r)\} r !\}+\frac{f^{(r+2)}(x)}{(r+2) !} n\left(\frac{(r+1)(r+2)}{2} x^{2}\right. \\
& \cdot \frac{n^{r} \Gamma(n / \gamma+r) \Gamma(n / \gamma-r+1)}{(n+\beta)^{r} \Gamma(n / \gamma+1) \Gamma(n / \gamma)} r !-x(r+2) \\
& \cdot\left\{\frac{n^{r+1} \Gamma(n / \gamma+r+1) \Gamma(n / \gamma-r)}{(n+\beta)^{r+1} \Gamma(n / \gamma+1) \Gamma(n / \gamma)}(r+1) ! x\right. \\
& +\frac{(r+1) n^{r} \Gamma(n / \gamma+r) \Gamma(n / \gamma-r)}{(n+\beta)^{r+1} \Gamma(n / \gamma+1) \Gamma(n / \gamma)}\{n r \\
& \left.\left.+\alpha\left(\frac{n}{\gamma}-r\right)\right\} r !\right\} \\
& +\frac{n^{r+2} \Gamma(n / \gamma+r+2) \Gamma(n / \gamma-r-1)}{(n+\beta)^{r+2} \Gamma(n / \gamma+1) \Gamma(n / \gamma)} \frac{(r+2) !}{2} x^{2} \\
& +\frac{(r+2) n^{r+1} \Gamma(n / \gamma+r+1) \Gamma(n / \gamma-r-1)}{(n+\beta)^{r+2} \Gamma(n / \gamma+1) \Gamma(n / \gamma)}\{n(r \\
& \left.+1)+\alpha\left(\frac{n}{\gamma}-r-1\right)\right\}(r+1) ! x \\
& +\frac{\alpha(r+1)(r+2) n^{r} \Gamma(n / \gamma+r) \Gamma(n / \gamma-r)}{(n+\beta)^{r} \Gamma(n / \gamma+1) \Gamma(n / \gamma)}\{n r \\
& \left.\left.+\frac{\alpha(n / \gamma-r)}{2}\right\} r !\right) .
\end{aligned}
$$

Now, the coefficients of $f^{(r)}(x), f^{(r+1)}(x)$, and $f^{(r+2)}(x)$ in the above expression tend to $r(\gamma(r-1)-\beta), r \gamma(1+2 x)+\alpha-\beta x$, and $x(1+\gamma x)$, respectively, which follows by using induction hypothesis on $r$ and taking the limit as $n \rightarrow \infty$. Hence, in order to prove (34), it is sufficient to show that $E_{2} \rightarrow 0$ as $n \rightarrow \infty$, which follows along the lines of the proof of Theorem 6 and by using Remark 2 and Lemmas 1 and 4 .

Remark 8. Particular case $\alpha=\beta=0$ was discussed in Theorem 4.1 in [4], which says that the coefficient of $f^{(r+1)}(x)$ converges to $r(1+2 \gamma x)$ but it converges to $r \gamma(1+2 x)$ and we get this by putting $\alpha=\beta=0$ in the above theorem.

Definition 9. The $m$ th order modulus of continuity $\omega_{m}(f, \delta$, $[a, b])$ for a function continuous on $[a, b]$ is defined by

$$
\begin{aligned}
& \omega_{m}(f, \delta,[a, b]) \\
& \quad=\sup \left\{\left|\Delta_{h}^{m} f(x)\right|:|h| \leq \delta ; x, x+h \in[a, b]\right\} .
\end{aligned}
$$

For $m=1, \omega_{m}(f, \delta)$ is usual modulus of continuity. 
Theorem 10. Let $f \in C_{\mu}[0, \infty)$ for some $\mu>0$ and $0<a<$ $a_{1}<b_{1}<b<\infty$. Then, for $n$ sufficiently large, one has

$$
\begin{aligned}
& \left\|\left(B_{n, \gamma}^{\alpha, \beta}\right)^{(r)}(f, \cdot)-f^{(r)}\right\|_{C\left[a_{1}, b_{1}\right]} \\
& \quad \leq M_{1} \omega_{2}\left(f^{(r)}, n^{-1 / 2},\left[a_{1}, b_{1}\right]\right)+M_{2} n^{-1}\|f\|_{\mu},
\end{aligned}
$$

where $M_{1}=M_{1}(r)$ and $M_{2}=M_{2}(r, f)$.

Proof. Let us assume that $0<a<a_{1}<b_{1}<b<\infty$. For sufficiently small $\eta>0$, we define the function $f_{\eta, 2}$ corresponding to $f \in C_{\mu}[a, b]$ and $t \in\left[a_{1}, b_{1}\right]$ as follows:

$$
f_{\eta, 2}(t)=\eta^{-2} \iint_{-\eta / 2}^{\eta / 2}\left(f(t)-\Delta_{h}^{2} f(t)\right) d t_{1} d t_{2}
$$

where $h=\left(t_{1}+t_{2}\right) / 2$ and $\Delta_{h}^{2}$ is the second order forward difference operator with step length $h$. For $f \in C[a, b]$, the functions $f_{\eta, 2}$ are known as the Steklov mean of order 2, which satisfy the following properties [11]:

(a) $f_{\eta, 2}$ has continuous derivatives up to order 2 over $\left[a_{1}, b_{1}\right]$.

(b) $\left\|f_{\eta, 2}^{(r)}\right\|_{C\left[a_{1}, b_{1}\right]} \leq \widehat{M_{1}} \eta^{-r} \omega_{2}(f, \eta,[a, b]), r=1,2$.

(c) $\left\|f-f_{\eta, 2}\right\|_{C\left[a_{1}, b_{1}\right]} \leq \widehat{M_{2}} \omega_{2}(f, \eta,[a, b])$.

(d) $\left\|f_{\eta, 2}\right\|_{C\left[a_{1}, b_{1}\right]} \leq \widehat{M_{3}}\|f\|_{\mu}$,

where $\widehat{M}_{i}, i=1,2,3$, are certain constants which are different in each occurrence and are independent of $f$ and $\eta$.

We can write by linearity properties of $B_{n, \gamma}^{\alpha, \beta}$,

$$
\begin{aligned}
\left\|\left(B_{n, \gamma}^{\alpha, \beta}\right)^{(r)}(f, \cdot)-f^{(r)}\right\|_{C\left[a_{1}, b_{1}\right]} \\
\leq\left\|\left(B_{n, \gamma}^{\alpha, \beta}\right)^{(r)}\left(f-f_{\eta, 2}, \cdot\right)\right\|_{C\left[a_{1}, b_{1}\right]} \\
\quad+\left\|\left(\left(B_{n, \gamma}^{\alpha, \beta}\right)^{(r)} f_{\eta, 2}, \cdot\right)-f_{\eta, 2}^{(r)}\right\|_{C\left[a_{1}, b_{1}\right]} \\
\quad+\left\|f^{(r)}-f_{\eta, 2}^{(r)}\right\|_{C\left[a_{1}, b_{1}\right]}:=P_{1}+P_{2}+P_{3} .
\end{aligned}
$$

Since $f_{\eta, 2}^{(r)}=\left(f^{(r)}\right)_{\eta, 2}(t)$, by property (c) of the function $f_{\eta, 2}$, we get

$$
P_{3} \leq \widehat{M_{4}} \omega_{2}\left(f^{(r)}, \eta,[a, b]\right) .
$$

Next, on an application of Theorem 7, it follows that

$$
P_{2} \leq \widehat{M_{5}} n^{-1} \sum_{i=r}^{r+2}\left\|f_{\eta, 2}^{(i)}\right\|_{C[a, b]} .
$$

Using the interpolation property due to Goldberg and Meir [12], for each $j=r, r+1, r+2$, it follows that

$$
\left\|f_{\eta, 2}^{(i)}\right\|_{C[a, b]} \leq \widehat{M_{6}}\left\{\left\|f_{\eta, 2}\right\|_{C[a, b]}+\left\|f_{\eta, 2}^{(r+2)}\right\|_{C[a, b]}\right\} .
$$

Therefore, by applying properties (c) and (d) of the function $f_{\eta, 2}$, we obtain

$$
P_{2} \leq \widehat{M_{7}} \cdot n^{-1}\left\{\|f\|_{\mu}+\delta^{-2} \omega_{2}\left(f^{(r)}, \mu,[a, b]\right)\right\} .
$$

Finally we will estimate $P_{1}$, choosing $a^{*}, b^{*}$ satisfying the conditions $0<a<a^{*}<a_{1}<b_{1}<b^{*}<b<\infty$. Suppose $\hbar(t)$ denotes the characteristic function of the interval $\left[a^{*}, b^{*}\right]$. Then

$$
\begin{aligned}
P_{1} & \leq\left\|\left(B_{n, \gamma}^{\alpha, \beta}\right)^{(r)}\left(\hbar(t)\left(f(t)-f_{\eta, 2}(t)\right), \cdot\right)\right\|_{C\left[a_{1}, b_{1}\right]} \\
& +\left\|\left(B_{n, \gamma}^{\alpha, \beta}\right)^{(r)}\left((1-\hbar(t))\left(f(t)-f_{\eta, 2}(t)\right), \cdot\right)\right\|_{C\left[a_{1}, b_{1}\right]} \\
& :=P_{4}+P_{5} .
\end{aligned}
$$

By Lemma 5, we have

$$
\begin{aligned}
\left(B_{n, \gamma}^{\alpha, \beta}\right)^{(r)}\left(\hbar(t)\left(f(t)-f_{\eta, 2}(t)\right), x\right) \\
=\frac{n^{r} \Gamma(n / \gamma+r) \Gamma(n / \gamma-r+1)}{(n+\beta)^{r} \Gamma(n / \gamma+1) \Gamma(n / \gamma)} \sum_{k=0}^{\infty} p_{n+\gamma r, k, \gamma}(x) \\
\quad \cdot \int_{0}^{\infty} b_{n-\gamma r, k+r, \gamma}(t) \hbar(t) \\
\quad \cdot\left(f^{(r)}\left(\frac{n t+\alpha}{n+\beta}\right)-f_{\eta, 2}^{(r)}\left(\frac{n t+\alpha}{n+\beta}\right)\right) d t .
\end{aligned}
$$

Hence,

$$
\begin{aligned}
& \left\|\left(B_{n, \gamma}^{\alpha, \beta}\right)^{r}\left(\hbar(t)\left(f(t)-f_{\eta, 2}(t)\right), \cdot\right)\right\|_{C\left[a_{1}, b_{1}\right]} \\
& \leq \widehat{M_{8}}\left\|f^{(r)}-f_{\eta, 2}^{(r)}\right\|_{C\left[a^{*}, b^{*}\right]} .
\end{aligned}
$$

Now, for $x \in\left[a_{1}, b_{1}\right]$ and $t \in[0, \infty) \backslash\left[a^{*}, b^{*}\right]$, we choose a $\delta>0$ satisfying $|(n t+\alpha) /(n+\beta)-x| \geq \delta$.

Therefore, by Lemma 4 and the Cauchy-Schwarz inequality, we have

$$
\begin{aligned}
I \equiv & \left(B_{n, \gamma}^{\alpha, \beta}\right)^{(r)}\left((1-\hbar(t))\left(f(t)-f_{\eta, 2}(t)\right), x\right), \\
|I| & \leq \sum_{\substack{2 i+j \leq r \\
i, j \geq 0}} n^{i} \frac{\left|Q_{i, j, r, \gamma}(x)\right|}{\{x(1+\gamma x)\}^{r}} \sum_{k=1}^{\infty} p_{n, k, \gamma}(x)|k-n x|^{j} \\
& \cdot \int_{0}^{\infty} b_{n, k, \gamma}(t)(1-\hbar(t)) \\
& \cdot\left|f\left(\frac{n t+\alpha}{n+\beta}\right)-f_{\eta, 2}\left(\frac{n t+\alpha}{n+\beta}\right)\right| d t \\
& +\frac{\Gamma(n / \gamma+r)}{\Gamma(n / \gamma)}(1+\gamma x)^{-n / \gamma-r}(1-\hbar(0)) \\
& \cdot\left|f\left(\frac{\alpha}{n+\beta}\right)-f_{\eta, 2}\left(\frac{\alpha}{n+\beta}\right)\right| .
\end{aligned}
$$


For sufficiently large $n$, the second term tends to zero. Thus,

$$
\begin{aligned}
|I| & \leq \widehat{M_{9}}\|f\|_{\mu} \sum_{\substack{i+j \leq j \leq r \\
i, j \geq 0}} n^{i} \sum_{k=1}^{\infty} p_{n, k, \gamma}(x)|k-n x|^{j} \\
& \cdot \int_{|t-x| \geq \delta} b_{n, k, \gamma}(t) d t \leq \widehat{M_{9}}\|f\|_{\mu} \delta^{-2 m} \sum_{\substack{2 i+j \leq r \\
i, j \geq 0}} n^{i} \\
& \cdot \sum_{k=1}^{\infty} p_{n, k, \gamma}(x)|k-n x|^{j}\left(\int_{0}^{\infty} b_{n, k, \gamma}(t) d t\right)^{1 / 2} \\
& \cdot\left(\int_{0}^{\infty} b_{n, k, \gamma}(t)\left(\frac{n t+\alpha}{n+\beta}-x\right)^{4 m} d t\right)^{1 / 2} \\
& \leq \widehat{M}_{9}\|f\|_{\mu} \delta^{-2 m} \sum_{2 i+j \leq r} n^{i} \\
& \cdot\left(\sum_{k=1}^{\infty} p_{n, k, \gamma}(x)|k-n x|^{2 j}\right)^{1 / 2}\left(\sum_{k=1}^{\infty} p_{n, k, \gamma}(x)\right. \\
& \left.\int_{0}^{\infty} b_{n, k, \gamma}(t)\left(\frac{n t+\alpha}{n+\beta}-x\right)^{4 m} d t\right)^{1 / 2} \cdot
\end{aligned}
$$

Hence, by using Remark 2 and Lemma 1, we have

$$
|I| \leq \widehat{M_{10}}\|f\|_{\mu} \delta^{-2 m} O\left(n^{(i+(j / 2)-m)}\right) \leq \widehat{M_{11}} n^{-q}\|f\|_{\mu}
$$

where $q=m-(r / 2)$. Now choosing $m>0$ satisfying $q \geq 1$, we obtain $I \leq \widehat{M_{11}} n^{-1}\|f\|_{\mu}$. Therefore, by property (c) of the function $f_{\eta, 2}(t)$, we get

$$
\begin{aligned}
P_{1} & \leq \widehat{M_{8}}\left\|f^{(r)}-f_{\eta, 2}^{(r)}\right\|_{C\left[a^{*}, b^{*}\right]}+\widehat{M_{11}} n^{-1}\|f\|_{\mu} \\
& \leq \widehat{M_{12}} \omega_{2}\left(f^{(r)}, \eta,[a, b]\right)+\widehat{M_{11}} n^{-1}\|f\|_{\mu} .
\end{aligned}
$$

Choosing $\eta=n^{-1 / 2}$, the theorem follows.

Remark 11. In the last decade the applications of $q$-calculus in approximation theory are one of the main areas of research. In 2008, Gupta [13] introduced $q$-Durrmeyer operators whose approximation properties were studied in [14]. More work in this direction can be seen in [15-17].

A Durrmeyer type $q$-analogue of the $B_{n, \gamma}^{\alpha, \beta}(f, x)$ is introduced as follows:

$$
\begin{aligned}
& B_{n, \gamma, q}^{\alpha, \beta}(f, x) \\
& =\sum_{k=1}^{\infty} p_{n, k, \gamma}^{q}(x) \int_{0}^{\infty / A} q^{-k} b_{n, k, \gamma}^{q}(t) f\left(\frac{[n]_{q} t+\alpha}{[n]_{q}+\beta}\right) d_{q} t \\
& \quad+p_{n, 0, \gamma}^{q}(x) f\left(\frac{\alpha}{[n]_{q}+\beta}\right),
\end{aligned}
$$

where

$$
\begin{aligned}
p_{n, k, \gamma}^{q}(x)= & q^{k^{2} / 2} \frac{\Gamma_{q}(n / \gamma+k)}{\Gamma_{q}(k+1) \Gamma_{q}(n / \gamma)} \\
& \cdot \frac{(q \gamma x)^{k}}{(1+q \gamma x)_{q}^{(n / \gamma)+k},} \\
b_{n, k, \gamma}^{q}(x)= & \gamma q^{k^{2} / 2} \frac{\Gamma_{q}(n / \gamma+k+1)}{\Gamma_{q}(k) \Gamma_{q}(n / \gamma+1)} \\
& \cdot \frac{(\gamma t)^{k-1}}{(1+\gamma t)_{q}^{(n / \gamma)+k+1},} \\
\int_{0}^{\infty} f(x) d_{q} x= & (1-q) \sum_{n=-\infty}^{\infty} f\left(\frac{q^{n}}{A}\right) \frac{q^{n}}{A}, \quad A>0 .
\end{aligned}
$$

Notations used in (53) can be found in [18]. For the operators (53), one can study their approximation properties based on $q$-integers.

\section{Conflict of Interests}

The authors declare that there is no conflict of interests regarding the publication of this research article.

\section{Acknowledgments}

The authors would like to express their deep gratitude to the anonymous learned referee(s) and the editor for their valuable suggestions and constructive comments, which resulted in the subsequent improvement of this research article.

\section{References}

[1] V. Gupta, D. K. Verma, and P. N. Agrawal, "Simultaneous approximation by certain Baskakov-Durrmeyer-Stancu operators," Journal of the Egyptian Mathematical Society, vol. 20, no. 3, pp. 183-187, 2012.

[2] D. K. Verma, V. Gupta, and P. N. Agrawal, "Some approximation properties of Baskakov-Durrmeyer-Stancu operators," Applied Mathematics and Computation, vol. 218, no. 11, pp. 6549-6556, 2012.

[3] V. N. Mishra, K. Khatri, L. N. Mishra, and Deepmala, "Inverse result in simultaneous approximation by Baskakov-DurrmeyerStancu operators," Journal of Inequalities and Applications, vol. 2013, article 586, 11 pages, 2013.

[4] V. Gupta, "Approximation for modified Baskakov Durrmeyer type operators," Rocky Mountain Journal of Mathematics, vol. 39, no. 3, pp. 825-841, 2009.

[5] V. Gupta, M. A. Noor, M. S. Beniwal, and M. K. Gupta, "On simultaneous approximation for certain Baskakov Durrmeyer type operators," Journal of Inequalities in Pure and Applied Mathematics, vol. 7, no. 4, article 125, 2006.

[6] V. N. Mishra and P. Patel, "Approximation properties of q-Baskakov-Durrmeyer-Stancu operators," Mathematical Sciences, vol. 7, no. 1, article 38, 12 pages, 2013. 
[7] P. Patel and V. N. Mishra, "Approximation properties of certain summation integral type operators," Demonstratio Mathematica, vol. 48, no. 1, pp. 77-90, 2015.

[8] V. N. Mishra, H. H. Khan, K. Khatri, and L. N. Mishra, "Hypergeometric representation for Baskakov-Durrmeyer-Stancu type operators," Bulletin of Mathematical Analysis and Applications, vol. 5, no. 3, pp. 18-26, 2013.

[9] V. N. Mishra, K. Khatri, and L. N. Mishra, "On simultaneous approximation for Baskakov Durrmeyer-Stancu type operators," Journal of Ultra Scientist of Physical Sciences, vol. 24, no. 3, pp. 567-577, 2012.

[10] W. Li, "The voronovskaja type expansion fomula of the modified Baskakov-Beta operators," Journal of Baoji College of Arts and Science (Natural Science Edition), vol. 2, article 004, 2005.

[11] G. Freud and V. Popov, "On approximation by spline functions," in Proceedings of the Conference on Constructive Theory Function, Budapest, Hungary, 1969.

[12] S. Goldberg and A. Meir, "Minimum moduli of ordinary differential operators," Proceedings of the London Mathematical Society, vol. 23, no. 3, pp. 1-15, 1971.

[13] V. Gupta, "Some approxmation properties of $q$-durrmeyer operators," Applied Mathematics and Computation, vol. 197, no. 1, pp. 172-178, 2008.

[14] A. Aral and V. Gupta, "On the Durrmeyer type modification of the q-Baskakov type operators," Nonlinear Analysis: Theory, Methods \& Applications, vol. 72, no. 3-4, pp. 1171-1180, 2010.

[15] G. M. Phillips, "Bernstein polynomials based on the $q$-integers," Annals of Numerical Mathematics, vol. 4, no. 1-4, pp. 511-518, 1997.

[16] S. Ostrovska, "On the Lupaş $q$-analogue of the Bernstein operator," The Rocky Mountain Journal of Mathematics, vol. 36, no. 5, pp. 1615-1629, 2006.

[17] V. N. Mishra and P. Patel, "On generalized integral Bernstein operators based on q-integers," Applied Mathematics and Computation, vol. 242, pp. 931-944, 2014.

[18] V. Kac and P. Cheung, Quantum Calculus, Universitext, Springer, New York, NY, USA, 2002. 


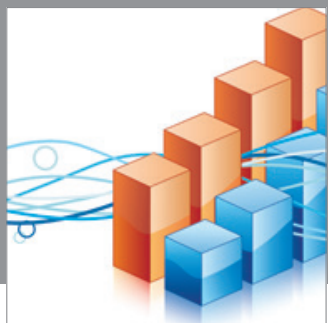

Advances in

Operations Research

mansans

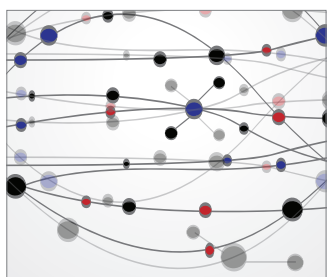

The Scientific World Journal
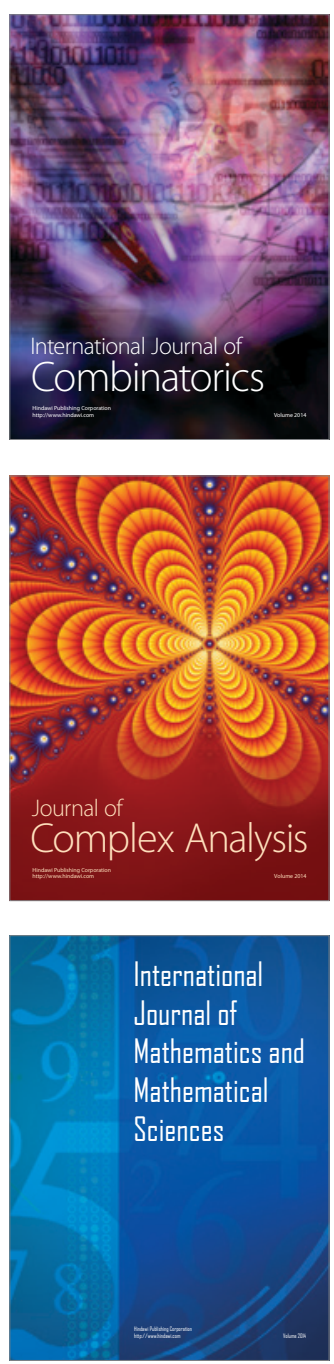
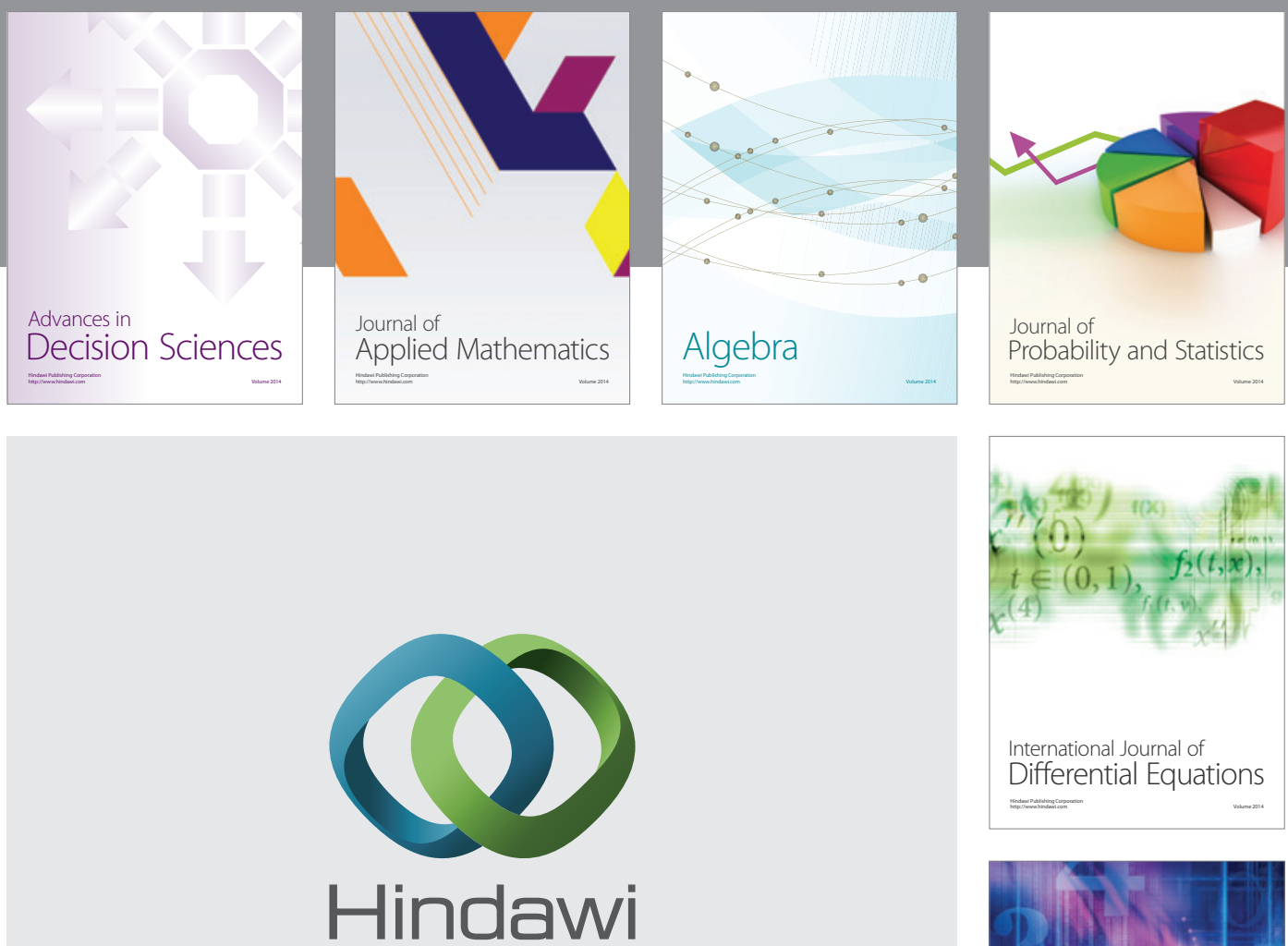

Submit your manuscripts at http://www.hindawi.com
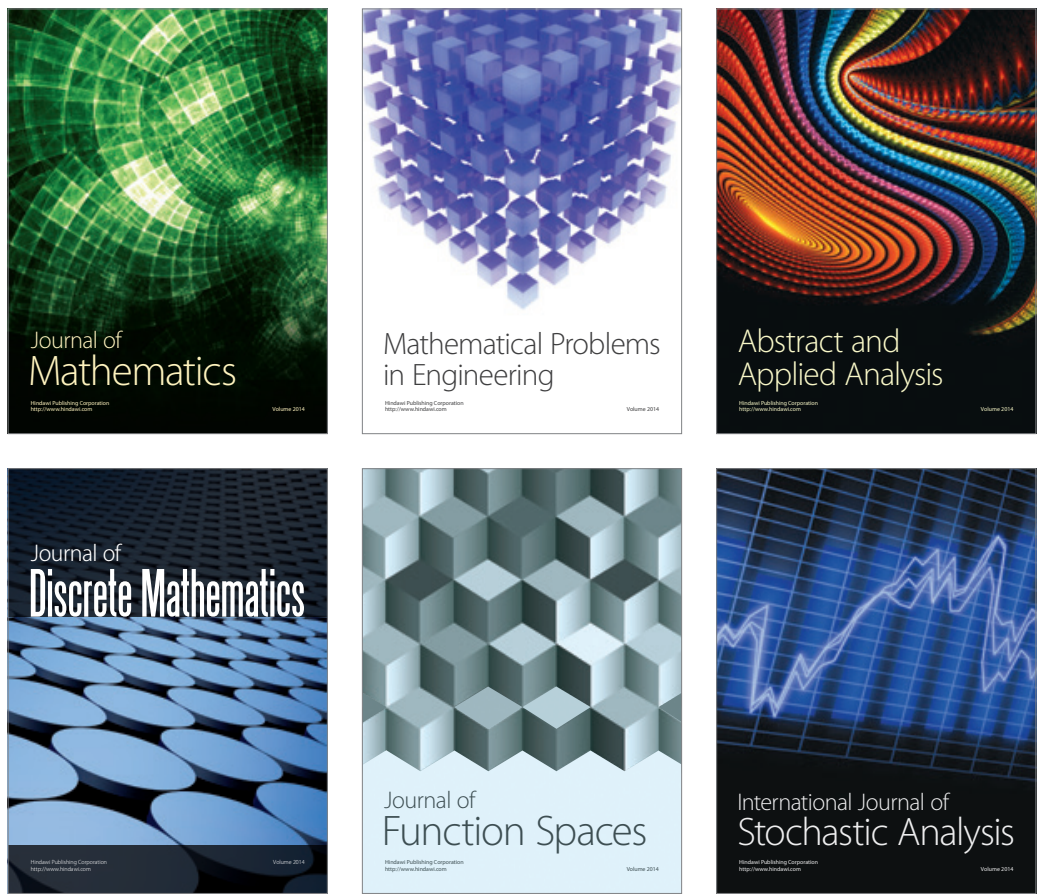

Journal of

Function Spaces

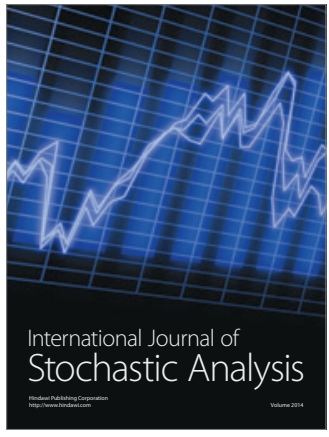

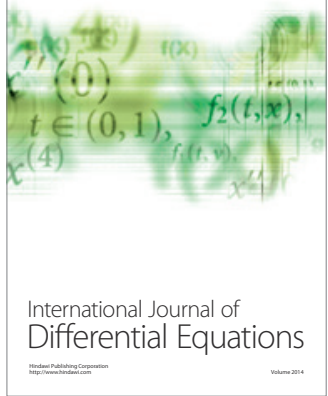
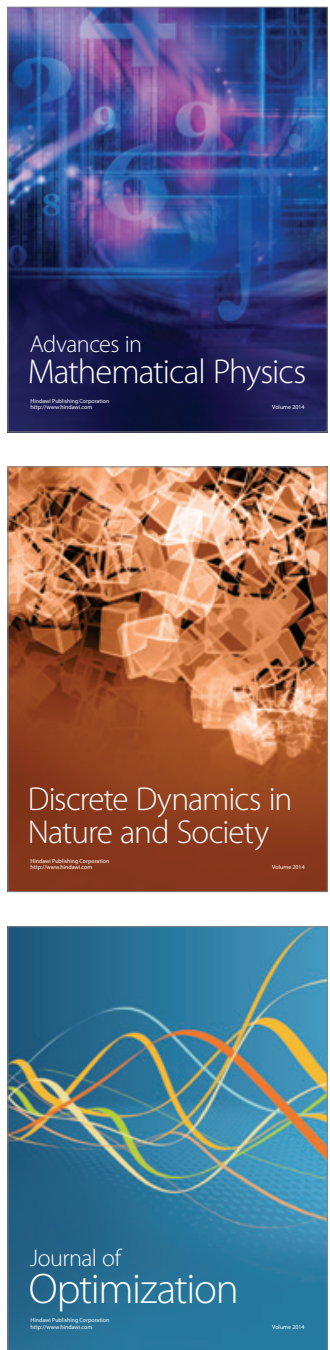